\title{
Multidimensional four-wave-mixing spectroscopy with squeezed light
}

Cite as: Appl. Phys. Lett. 116, 244001 (2020); https://doi.org/10.1063/5.0009575

Submitted: 04 April 2020 . Accepted: 01 June 2020. Published Online: 19 June 2020

(iD) Zhenquan Yang, (iD) Prasoon Saurabh, (iD) Frank Schlawin, (D) Shaul Mukamel, and (i) Konstantin E. Dorfman

\section{COLLECTIONS}

Paper published as part of the special topic on Quantum Sensing with Correlated Light Sources
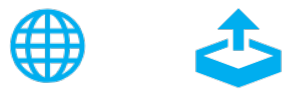

\section{ARTICLES YOU MAY BE INTERESTED IN}

Interferometric two-photon-absorption spectroscopy with three entangled photons

Applied Physics Letters 116, 174003 (2020); https://doi.org/10.1063/5.0004617

Squeezed light induced two-photon absorption fluorescence of fluorescein biomarkers

Applied Physics Letters 116, 254001 (2020); https://doi.org/10.1063/5.0010909

How many photons does it take to form an image?

Applied Physics Letters 116, 260504 (2020); https://doi.org/10.1063/5.0009493

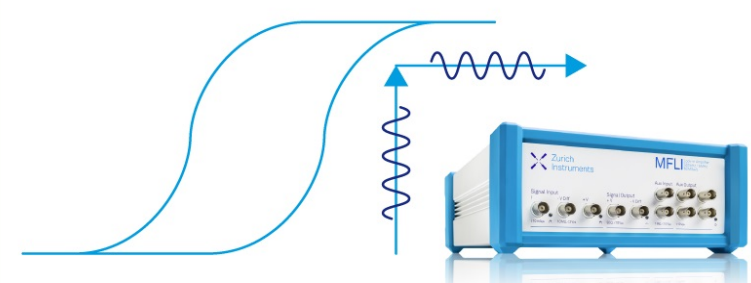

Webinar

How to Characterize Magnetic

Materials Using Lock-in Amplifiers

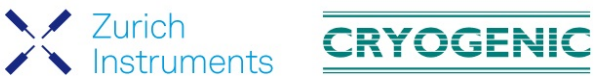




\title{
Multidimensional four-wave-mixing spectroscopy with squeezed light
}

\author{
Cite as: Appl. Phys. Lett. 116, 244001 (2020); doi: 10.1063/5.0009575 \\ Submitted: 4 April 2020 - Accepted: 1 June 2020 . \\ Published Online: 19 June 2020

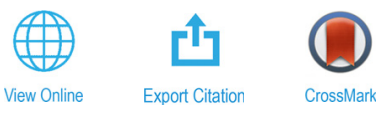

Zhenquan Yang, ${ }^{1}$ (D) Prasoon Saurabh, ${ }^{1}$ (D) Frank Schlawin, ${ }^{2}$ (D) Shaul Mukamel, ${ }^{3}$ (D) and Konstantin E. Dorfman ${ }^{7, a)}$ (iD

AFFILIATIONS

${ }^{7}$ State Key Laboratory of Precision Spectroscopy, East China Normal University, Shanghai 200062, China

${ }^{2}$ Department of Physics, University of Oxford, Oxford OX1 1PU, United Kingdom

${ }^{3}$ Chemistry Department and Physics and Astronomy Department, University of California, Irvine, California 92697-2025, USA

Note: This paper is part of the APL Special Collection on Quantum Sensing with Correlated Light Sources

a) Author to whom correspondence should be addressed: dorfmank@lps.ecnu.edu.cn

\begin{abstract}
A set of nonlinear spectroscopic measurements with quantum light is presented. It involves a strong pump with frequency $\omega_{p u}$ and a weak probe at $\omega_{p r}$ interacting with a solid state target to generate a conjugated beam $\omega_{c}=2 \omega_{p u}-\omega_{p r}$ via nondegenerate four-wave mixing. The $\chi^{(3)}$ susceptibility can be measured by the noise spectra of the intensity difference of the squeezed beams. We discuss three spectroscopic setups based on squeezed light: first, single four-wave mixing in a single crystal; second, cascading scheme involving two crystals; third, an $S U(1,1)$ interferometer based on two separate four-wave mixing processes. We further investigate the microscopic noise and optical losses in all three setups. Simulations are presented for silicon-vacancy color centers in diamond.
\end{abstract}

Published under license by AIP Publishing. https://doi.org/10.1063/5.0009575

Four wave mixing (FWM) is a nonlinear optical process that underlies diverse applications to spectroscopy, photonic quantum computation, and communication. In spectroscopy, three photon fields drive electronic transitions, giving rise to the fourth signal field, satisfying momentum and energy conservation. In Raman quantum memories, ${ }^{1-4}$ several pulses are used to store information in the system, which is then retrieved by another pulse. FWM is also used to generate entangled photons $s^{5}$ or squeezed light ${ }^{6-8}$ pulses with applications to quantum information processing, ${ }^{9}$ imaging, ${ }^{10}$ and sensing. ${ }^{11}$ Strong quantum correlations of light fields created by FWM have been demonstrated in hot alkali vapors ${ }^{12-14}$ and solid state systems. ${ }^{15}$ Recent progress in nonlinear spectroscopy with nonclassical light ${ }^{16-18}$ demonstrates the use of quantum light sources to study the matter response. ${ }^{19}$ FWM-generated light carries information about the material system through its third order susceptibility $\chi^{(3)}$ given by Eq. (1). It can be detected using either classical transmission spectra given by Eq. (4) or the quantum measurement via the relative squeezing spectra given by Eq. (5). Squeezed light beams allow us to perform high precision measurements beyond the shot-noise limit, which is especially important in the case of weakly absorbing materials. In the standard quantum optics treatment of squeezed light generation, the material susceptibility is taken as a frequency-independent prefactor. This is justified when all fields are far off-resonant with respect to matter.
Resonant nonlinear spectroscopy investigates material properties, which requires an explicit treatment of the matter response via frequency-dependent nonlinear susceptibilities. We shall discuss the three setups shown in Fig. 1, which are based on a single FWM (a), two FWMs in a cascade (b), and $S U(1,1)$ interferometers (c).

We focus on the four-level double-lambda scheme with levels $g$, $s, e$, and $f$ [see Fig. 1(d)]. The third-order susceptibility that governs FWM is derived by third-order perturbation expansion in the radiation/matter coupling represented by a set of Feynman diagrams given in Fig. S1 of supplementary material, which includes four terms,

$$
\chi^{(3)}\left(-\omega_{p r},-\omega_{c} ; 2 \omega_{p u}\right)=\sum_{p=a, b, q=I, I I} \chi_{p q}^{(3)}\left(-\omega_{p r},-\omega_{c} ; 2 \omega_{p u}\right) .
$$

The two "a" terms are

$$
\chi_{a I}^{(3)}=\sum_{j, k=e, f} \frac{\mu_{j g} \mu_{k g}^{*} \mu_{j s} \mu_{k s}^{*}}{\Delta_{k g}^{\prime} \Delta_{s g} \Delta_{j g}}, \quad \chi_{a I I}^{(3)}=\sum_{j, k=e, f} \frac{\mu_{j g} \mu_{k g}^{*} \mu_{j s} \mu_{k s}^{*}}{\Delta_{k g}^{* *} \Delta_{s g}^{*} \Delta_{j g}},
$$

where $\Delta_{j g}=\omega_{p u}-\omega_{j g}+i \gamma_{j g}, \Delta_{s g}=\omega_{p u}-\omega_{p r}-\omega_{s g}+i \gamma_{s g}$, and $\Delta_{j g}^{\prime}=2 \omega_{p u}-\omega_{p r}-\omega_{j g}+i \gamma_{j g}, j=e, f$. Contributions of diagrams $b I$ and $b I I$ can be obtained by interchanging $\omega_{p r} \rightarrow \omega_{c}$ and $\Delta_{s g}$ $\rightarrow \Delta_{g s}$, where $\Delta_{g s}=\omega_{p u}-\omega_{c}-\omega_{g s}+i \gamma_{g s} . \gamma_{m n}, m, n=g, s, e$ are the 


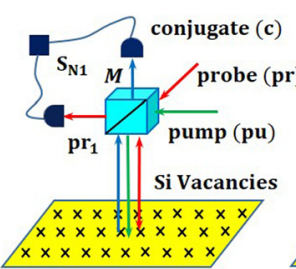

(a)

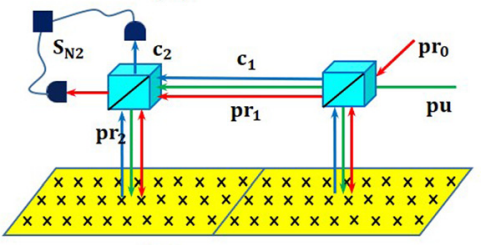

(c)

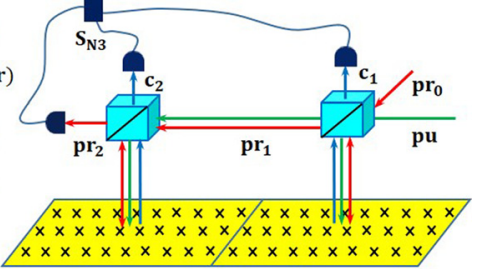

(b)

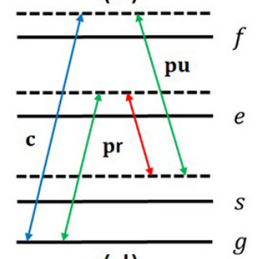

(d)
FIG. 1. Schematic of the proposed measurements. (a) Single FWM, (b) cascaded two FWMs, (c) SU $(1,1)$ interferometer, and (d) four-level diagram for the FWM in the $\mathrm{SiV}^{-}$centers in diamond. Parameters used in the simulations are summarized in the supplementary material.

dephasing rates of the dipole transitions, and the energy conservation $\omega_{p r}+\omega_{c}=2 \omega_{p u}$ is explicitly taken into account. Contributions of diagrams $i, i$, and $i i$ are accounted by permutations of $j$ and $k$ in the expressions [see Eq. (S3) of the supplementary material].

The setup for a single FWM experiment is shown in Fig. 1(a). A strong pump beam with frequency $\omega_{p u}$ and a weak probe beam with frequency $\omega_{p r}$ mix at the surface of the solid state platform. A conjugate beam at frequency $\omega_{c}=2 \omega_{p u}-\omega_{p r}$ is generated by the FWM process and is collected together with the reflected probe beam on a frequency mixer. The selected two wavelengths are finally sent into the two arms of the interferometer. Two detectors measure intensity of each (probe and conjugate) as well as their relative noise (squeezing) via balanced homodyne detection. While the input pump and the probe fields are classical, the amplified probe and the generated conjugate beams have quantum correlations. Detection of the output-probe and conjugate fields in coincidence reveals the material response governed by a susceptibility (1), which depends on the energies of the four eigenstates in Fig. 1(d) and their dephasing rates. It has been demonstrated that squeezing can manifest when the FWM is off-resonant with the excited states (e.g., $e$ and $f$ ) since resonant electronic excitation results in absorption losses rather than gain. ${ }^{12}$ Following the standard treatment of squeezing, ${ }^{20}$ we use the input-output relations in Eqs. (S4) and (S5) of the supplementary material for the probe and conjugate beams. Using the number operator for the incident probe $\hat{N}_{0}=\hat{a}_{p r}^{(i n) \dagger} \hat{a}_{p r}^{(i n)}$, we shall calculate the output photon numbers

$$
\begin{aligned}
\left\langle\hat{N}_{p r}\left(\omega_{p r}, \omega_{p u}\right)\right\rangle & \simeq G\left(-\omega_{p r},-\omega_{c} ; 2 \omega_{p u}\right)\left\langle\hat{N}_{0}\right\rangle, \\
\left\langle\hat{N}_{c}\left(\omega_{p r}, \omega_{p u}\right)\right\rangle & \simeq\left[G\left(-\omega_{p r},-\omega_{c} ; 2 \omega_{p u}\right)-1\right]\left\langle\hat{N}_{0}\right\rangle,
\end{aligned}
$$

where

$$
G\left(-\omega_{p r},-\omega_{c} ; 2 \omega_{p u}\right)=\cosh ^{2}\left[\left|A \chi^{(3)}\left(-\omega_{p r},-\omega_{c} ; 2 \omega_{p u}\right)\right|\right]
$$

is the FWM gain, $A \propto \mathscr{E}_{p u}^{2} L$ is the normalization constant, $L$ is a propagation length inside the crystal, and $\mathscr{E}_{p u}$ is the classical amplitude of the pump beam. Thus, by measuring the probe intensity, one can directly detect $\chi^{(3)}$. $^{21}$

In addition to the FWM gain, one can measure the noise figure (NF) of the intensity difference that characterizes the degree of squeezing of the probe and the conjugate fields. It is defined as the relative intensity noise divided by the sum of the shot-noise figures of the two beams,

$$
S_{N}\left(\omega_{p r}, \omega_{p u}\right)=\frac{\operatorname{Var}\left(\hat{N}_{p r}-\hat{N}_{c}\right)}{\left\langle\hat{N}_{p r}\right\rangle+\left\langle\hat{N}_{c}\right\rangle}=\frac{1}{2 G\left(-\omega_{p r},-\omega_{c} ; 2 \omega_{p u}\right)-1},
$$

where $G$ is given by Eq. (4). $S_{N}<1$ indicates quantum squeezing, whereas $S_{N}>1$ represents classical correlations. Note that in the absence of additional sources of noise, both gain $G$ and squeezing $S_{N}$ provide equivalent measures of the susceptibility $\chi^{(3)}$. Below, we show that this is not the case once additional noise in detection or imperfect transmission is taken into account.

Closer to material resonances, other processes may compete with the FWM and reduce the optical gain. For instance, spontaneous emission from either $e$ or $f$ to state $s$ initiated by a pump photon or a similar process with final state $g$ can give rise to a pair of photons with the same energy of the probe and conjugate beams. These photons are indistinguishable by their frequencies, and since they have not been generated by FWM, they are not phase matched and, therefore, uncorrelated and constitute a quantum noise. To describe such noise contributions, one typically uses Langevin theory. ${ }^{20}$ While this level of theory certainly helps quantify phenomenologically the effects of noise, spectroscopic applications will require a microscopic theory of the noise induced optical losses. Including noise, the input/output relation for the field operators now reads ${ }^{20}$

$$
\begin{gathered}
\hat{a}_{p r} \rightarrow \sqrt{\eta_{p r}} \hat{a}_{p r}+\sqrt{1-\eta_{p r}} \hat{x}_{p r}, \\
\hat{a}_{c} \rightarrow \sqrt{\eta_{c}} \hat{a}_{c}+\sqrt{1-\eta_{c}} \hat{x}_{c},
\end{gathered}
$$

where $\eta_{k}=\cos \left(r_{k}\right)^{2}, k=p r, c$ is a loss amplitude due to the noise, which represents the fraction of the field amplitude transmitted through the sample. $2 B_{k} \chi_{k}^{(1)}\left(-\omega_{k}, \omega_{p u}\right)=r_{k} e^{i \phi_{k}}$ is a susceptibility due to the noise, where both $r_{k}=\left|\zeta_{k}\right|$ and $\phi_{k}$ are real functions, $k=p r, c$. $B_{k} \sim L$ is a normalization constant. The susceptibilities $\chi_{p r}^{(1)}$ and $\chi_{c}^{(1)}$ are given by

$$
\chi_{p r}^{(1)}=\sum_{j=e, f} \frac{\mu_{j s} \mu_{j g}^{*}}{\Delta_{s g} \Delta_{j g}}, \quad \chi_{c}^{(1)}=\sum_{j=e, f} \frac{\mu_{j g} \mu_{j s}^{*}}{\Delta_{s g}\left(\Delta_{j g}^{\prime}-\Delta_{s g}\right)} .
$$

The noise operators $\hat{x}_{p r}$ and $\hat{x}_{c}$ satisfy the boson commutation rules $\left[\hat{x}_{j}, \hat{x}_{j}^{\dagger}\right]=1$. Using Eq. (6), we obtain for the average photon number,

$$
\begin{aligned}
\left\langle\hat{N}_{p r}\left(\omega_{p u}, \omega_{p r}\right)\right\rangle & \simeq \eta_{p r}\left(-\omega_{p r}, \omega_{p u}\right) G\left(-\omega_{p r},-\omega_{c} ; 2 \omega_{p u}\right)\left\langle\hat{N}_{0}\right\rangle, \\
\left\langle\hat{N}_{c}\left(\omega_{p r}, \omega_{p u}\right)\right\rangle & \simeq \eta_{c}\left(-\omega_{c}, \omega_{p u}\right)\left[G\left(-\omega_{p r},-\omega_{c} ; 2 \omega_{p u}\right)-1\right]\left\langle\hat{N}_{0}\right\rangle,
\end{aligned}
$$

and the FWM gain is given by Eq. (4). The NF is now given by

$$
S_{N}=1+\frac{2(G-1)\left(G\left(\eta_{p r}-\eta_{c}\right)^{2}-\eta_{c}^{2}\right)}{\eta_{p r} G+\eta_{c}(G-1)} .
$$

Note that for a sufficiently large gain $G$, noise can make $S_{N}>1$ for $\eta_{p r} \neq \eta_{c}$. However, if the noise in both quantum-correlated beams is 
balanced such that optical loss amplitudes are identical $\eta_{p r}=\eta_{c}=\eta$, then the quantum correlation persists

$$
S_{N}=1-\eta \frac{2(G-1)}{2 G-1}<1 .
$$

Balanced losses can be achieved if the susceptibilities $\chi_{c}^{(1)}$ and $\chi_{p r}^{(1)}$ are the same. Note that a FWM with noise is essentially a $\chi^{(5)}$ process where we have factorized a pure FWM governed by $\chi^{(3)}$ from the noise described by $\chi^{(1)}$, where the latter describes the losses after FWM. While it looks like the balanced losses, it always yields higher squeezing degree if $\eta$ is independent of frequencies according to Eq. (10). However, simulations of the noise spectrum may yield the opposite result in certain frequency regimes.

The following simulations use the four-level system shown in Fig. 1(d), which represents the diamond surface containing an ensemble of the silicon-vacancy color centers ( $\mathrm{SiV}^{-}$). The details of the $a b$ initio theory used for the simulations along with simulation parameters are given in the supplementary material. The simulated photoluminescence spectrum shown in Fig. 2(a) agrees with experiment, ${ }^{22}$ and has four features. ${ }^{23,24}$ Figure 2(b) shows the Gain spectrum given by Eq. (8) for the probe field for a fixed pump frequency. For $\omega_{p u}=\omega_{1} \equiv \omega_{f s}$, which will hereafter be referred to as a "low frequency," there are two weak closely spaced energy cross-peaks between $\omega_{p r_{1}}=2 \omega_{1}-\omega_{f g}$ and $\omega_{p r_{1}}=\omega_{1}-\omega_{s g}$. Similarly at $\omega_{p u}=\omega_{2}$ ("high frequency"), the three stronger peaks are one cross-peak between $\omega_{p r_{2}}=$ $\omega_{e s}, \omega_{p r_{2}}=\omega_{2}-\omega_{s g}$ and two peaks at $\omega_{p r_{3}}=2 \omega_{2}-\omega_{f g}$ and $\omega_{p r_{4}}=2 \omega_{2}-\omega_{e g}$. It follows that optical losses reduce the gain according to Eq. (8) such that the frequency gain at the cross-peak with $\omega_{p r 2}$ disappears and the losses indicated by $G<1$ in the green curve in Fig. 2(b) appear instead, while the low frequency features at $\omega_{p r 1}$ and the high frequency peak at $\omega_{p r_{3}}$ and $\omega_{p r_{4}}$ remain almost intact as depicted by the red and green curves, respectively. These observations are a consequence of the noise spectra displayed

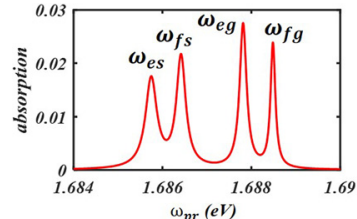

(a)

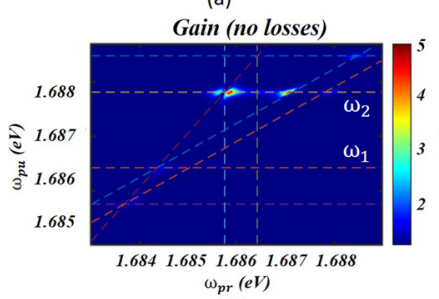

(c)

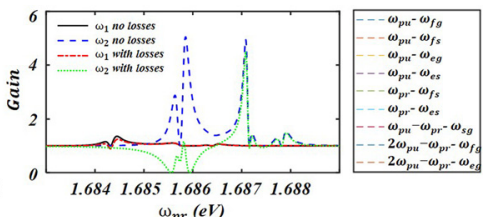

(b)

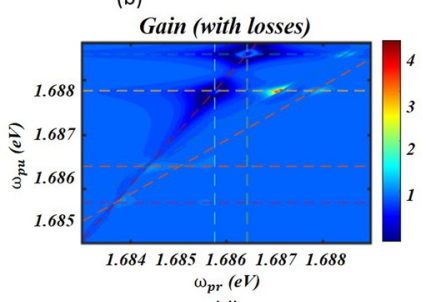

(d)
FIG. 2. (a) The simulated photoluminescence excitation spectrum at $T=5 \mathrm{~K}$ of the Si-vacancy ensemble, (b) 1D probe cite gain spectrum given by Eq. (3) for a single FWM vs the probe frequency at two different values for the fixed pump frequency $\omega_{\text {pu }}=\omega_{1,2}$, where $\omega_{1}=1.686415 \mathrm{eV}$ and $\omega_{2}=1.6877 \mathrm{eV}$. (c) and (d) $2 \mathrm{D}$ probe gain spectrum vs $\omega_{p u}$ and $\omega_{p r}$ without and with optical losses [see Eq. (8) at $\left.B_{p r}=0.01 A\right]$, respectively. The dashed lines mark resonances indicated in the inset of Fig. 3(b). The gain normalization constant in Eq. (1) is $A=1.735$. in Fig. 2S(a) of the supplementary material, where in the vicinity of the peaks at $\omega_{p r 1}, \omega_{p r 3}$, and $\omega_{p r 4}$, the losses are negligible. On the other hand, the cross-peak at $\left(\omega_{p r_{2}}, \omega_{2}\right)$ has strong noise, which significantly reduces the signal. The apparent symmetry of the noise spectra with respect to $\omega_{p u}$ and $\omega_{p r}$ arises due to the fact that spontaneous emission into the probe photon occurs at the nearly equal pump energy, which corresponds to the noise affecting primarily diagonal $\omega_{p u} \sim \omega_{p r}$. Since two out of four peaks in Fig. 2(b) are cross-peaks, it is convenient to depict spectra as a $2 \mathrm{D}$ spectrogram [see Figs. 2(c) and 2(d)], which are commonly used in photon echo time-domain measurements. ${ }^{25}$ The FWM considered here is a CW frequency-domain technique; however, the $2 \mathrm{D}$ spectrograms can be interpreted similar to those of the photon echo. Four horizontal dashed lines at $\omega_{p u}=\omega_{e g}, \omega_{f g}, \omega_{e s}, \omega_{f s}$ and two vertical dashed lines at $\omega_{p r}=\omega_{e s}, \omega_{f s}$ represent single-photon resonances for the pump and probe fields, respectively. Three diagonal dashed lines correspond to the two-photon resonances: $\omega_{p u}-\omega_{p r}=\omega_{s g}, 2 \omega_{p u}-\omega_{p r}$ $=\omega_{e g}$, and $2 \omega_{p u}-\omega_{p r}=\omega_{f g}$. The absence of off diagonal peaks indicates no coherent superposition between $f$ and $e$ states due to the narrow bandwidth of the applied fields. These resonances have been already discussed in Figs. 2(a) and 2(b). The contour plot color bar in Figs. 2(c) and 2(d) indicates a similar drop of the maximum intensity from $G \sim 5$ down to zero at $\omega_{p r_{2}}$ due to the strong noise, while gain remains intact for other resonances at $\omega_{p r_{1}}, \omega_{p r_{3}}$, and $\omega_{p r_{4}}$.

We next turn to the NF given by Eq. (5). Similar to the gain shown in Fig. 2(b) for a fixed $\omega_{p u}=\omega_{j}, j=1,2$, the NF shown in Fig. 3(a) has four peaks corresponding to $\omega_{p r_{k}}, k=1,2,3,4$. This can be seen more clearly from the 3D plot shown in Fig. 3(b), which shows that the squeezing almost vanishes away from the resonances corresponding to the blue and solid black curves in Fig. 3(a). The unbalanced optical losses due to the noise affect the peaks at $\omega_{p r 1}$ and $\omega_{p r 2}$, while peaks at $\omega_{p r 3}$ and $\omega_{p r_{4}}$ are affected only slightly, as indicated by red and dotted black curves in Fig. 3(a). This follows directly from the noise spectrum, which has a complex spectral dependence, overlapping with the $\chi^{(3)}$ resonance structure (see Fig. S2 of the

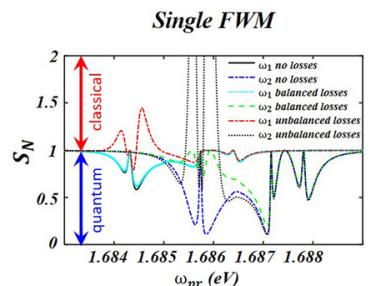

$\omega_{p r}(e)$
(a)

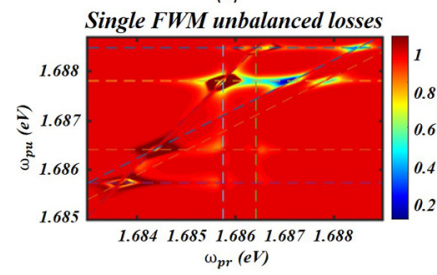

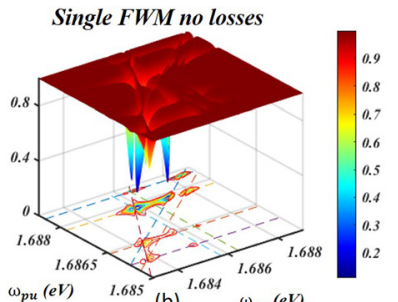

$\omega_{p u}(\mathrm{eV}) \quad{ }^{1.685}(\mathrm{~b}) \omega_{\mathrm{pr}}(\mathrm{eV})$

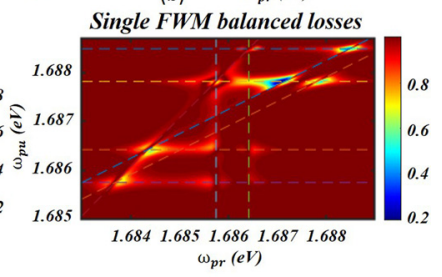

(d)
FIG. 3. (a) The same as Fig. 2(b) but for the 1D NF given by Eq. (5). (b) 3D spectra of the NF vs $\omega_{p u}$ and $\omega_{p r}$. (c) and (d) NF with unbalanced $\left(\eta_{p r} \neq \eta_{c}\right)$ and balanced $\left(\eta_{p r}=\eta_{c}\right)$ losses, respectively. The rest of the parameters are the same as in Fig. 2. The noise normalization used is $B_{p r}=B_{c}=0.02$. 
supplementary material). Figure 3(c) shows the NF given by Eq. (9) where unbalanced losses result in the classical regime $S_{N}>1$ for resonances $\omega_{p r 1}$ and $\omega_{p r 2}$ along the main diagonal $\omega_{p u}-\omega_{p r}=\omega_{s g}$, while the quantum regime $S_{N}<1$ is observed for $\omega_{p r_{3}}$ and $\omega_{p r_{4}}$ along diagonals $\omega_{p r}=2 \omega_{p u}-\omega_{e g}$ and $\omega_{p r}=2 \omega_{p u}-\omega_{f g}$. In addition to the four peaks resolved by the gain measurement, the three new cross-peaks show up between $\omega_{p u}=\omega_{e s}$ and $\omega_{p r 5}=\omega_{p u}-\omega_{s g}, \omega_{p u}=\omega_{f g}$ and $\omega_{p r 6}=\omega_{p u}-\omega_{s g}$, and $\omega_{p r 7}=2 \omega_{p u}-\omega_{f g}$. The emergence of new well-resolved resonances clearly demonstrates the benefits of quantum $S_{N}$ measurements over the classical gain detection. The maximum squeezing also changes from 0 to 0.2 , showing that the noise reduces the degree of quantum correlations. The corresponding case with balanced losses Eq. (10) shown in Fig. 3(d) shows an extended regime of the quantum correlations along the main diagonal $\omega_{p u}-\omega_{p r}=\omega_{s g}$. For instance, in the vicinity of the $\omega_{p r 1}$ and $\omega_{p r 2}$ cross-peak, balanced losses yield 0.5 vs 1 for the unbalanced case indicated by the turquoise line in Fig. 3(a).

One can further improve the detection of quantum correlations by cascading multiple FWM processes. For instance, one can allow the pump and probe beams to pass through another material sample initiating the second FWM, while the conjugate beam from the first FWM serves as a reference [see Fig. 1(b)]. Together with the conjugate beam from the first FWM, the output probe and conjugate from the second FWM constitute a triple output, which can show squeezing between all three beams. The input-output relation of the cascaded two FWM scheme is given by Eqs. (S8) and (S9) of the supplementary material. The output squeezing between the three beams is then given by: ${ }^{12,14}$

$$
S_{N 3}=\frac{\operatorname{Var}\left(\hat{N}_{p r 2}-\hat{N}_{c 2}-\hat{N}_{c 1}\right)}{\left\langle\hat{N}_{p r 2}\right\rangle+\left\langle\hat{N}_{c 2}\right\rangle+\left\langle\hat{N}_{c 1}\right\rangle}=\frac{1}{2 G_{2} G_{1}-1} .
$$

Note that for moderate to high gain, the triple squeezing can be substantially higher than that for a single FWM. This has been confirmed experimentally. ${ }^{14}$ If both FWM are identical $G_{1}=G_{2}=G$, we have $S_{N 3}=\left(2 G^{2}-1\right)^{-1}$. Figure 4 (a) shows that two cascaded FWM
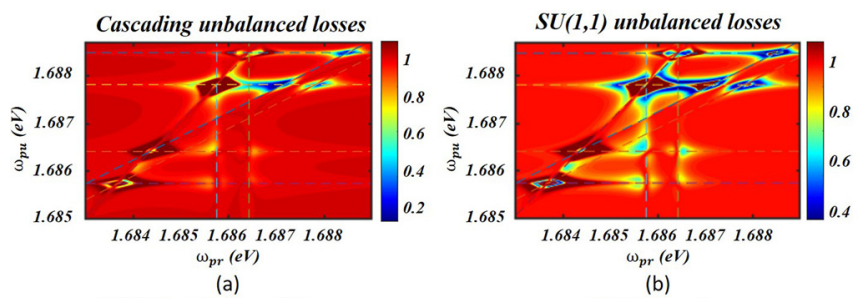

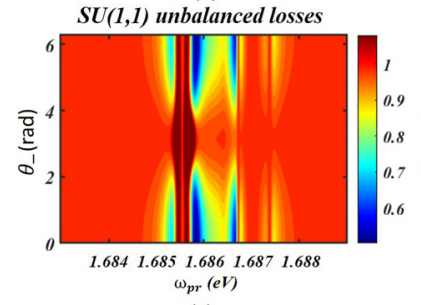

(c)

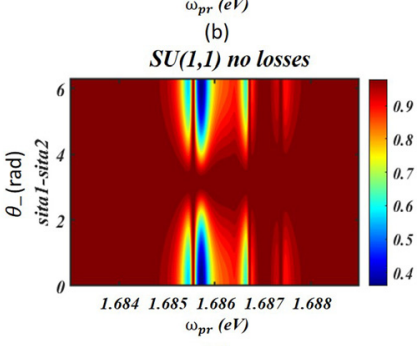

(d)
FIG. 4. Top: comparison of the 2D NFs between cascading $S_{N 3}$ given by Eq. (11) (a) and $S U(1,1) S_{N 2}$ given by Eq. (12) for identical cells. Bottom: effects of the squeezing phase in Eq. (12) vs $\omega_{p r}$ at fixed $\omega_{p u}=1.68705 \mathrm{eV}$. The rest of the parameters are the same as in Fig. 2. subjected to unbalanced noise have slight improvement compared to the single FWM shown in Fig. 3(c). This is because spectral features corresponding to $S_{N}<1$ become more pronounced, indicating that spectral resolution is improved from the single FWM to the cascading scheme and is further enhanced in the $S U(1,1)$ setup. The corresponding expression for the balanced losses case is given in Eq. (S52) of the supplementary material.

We now turn to the $S U(1,1)$ interferometer ${ }^{26}$ shown in Fig. 1(c), whose operation can be viewed as a transformation under the Lorenz group. ${ }^{27}$ After the first FWM, both probe and conjugate beams are directed into the second FWM and generate another probe-conjugate squeezed output. This arrangement can be used for interferometry and has been shown to yield a higher contrast than, e.g., Mach-Zehnder interferometer. ${ }^{13}$ The input-output relation of the cascaded two FWM scheme is given by Eqs. (S15) and (S16) of the supplementary material. This results in the NF for the second FWM, which yields:

$$
S_{N 2}=\frac{1}{\left(2 G_{1}-1\right)\left(2 G_{2}-1\right)+4 \sqrt{G_{1} G_{2}\left(G_{1}-1\right)\left(G_{2}-1\right)} \cos \theta_{-}},
$$

where $\theta_{-}=\theta_{2}-\theta_{1}$. For identical FWMs $G_{1}=G_{2}=G, \theta_{-}=0$, this reduces to $S_{N 2}=\left[2(2 G-1)^{2}-1\right]^{-1}<S_{N}$, which yields higher squeezing compared to a single FWM. Note that unlike the cascading scheme, the squeezing in the $S U(1,1)$ interferometer depends on the squeezing phase, which makes it suitable for interferometric measurements.

Figure 4(b) shows that $S U(1,1)$ with unbalanced losses yields a lower maximum degree of quantum correlations compared to cascading $(0.4 \mathrm{vs} 0.2)$. At the same time, in the vicinity of the resonances at $\omega_{p r_{j}}, j=1-7$, quantum correlations emerge in the larger vicinity of cross-peaks upon squeezing down to 0.4 . We can further investigate the squeezing phase dependence in the $S U(1,1)$ interferometer. Figure 4 (c) shows that quantum squeezing occurs in the vicinity of $\omega_{p r}=\omega_{e s}, \omega_{f s}$, and $\omega_{p r 3}$ manifested as a double-dip structure with a peak (with $S_{N}>1$ ) at the exact resonance for the entire range of $\theta_{-}$ from 0 to $2 \pi$ except in the vicinity of $\theta_{-}=\pi$. The resonance linewidth decreases from 0 to $\pi$ and increases from $\pi$ to $2 \pi$. The maximum degree of quantum squeezing reaches $\sim 0.5$. The corresponding expressions are given in Eq. (S59) of the supplementary material. Figure 4 (d) shows that in the absence of noise, the double-dip structure becomes narrower at the exact resonance with quantum squeezing $\sim 0.4$, while the area around $\theta_{-}=\pi$ with $S_{N}>$ 1 is reduced as well. Thus, adjusting the squeezing phase can further improve spectral resolution of the quantum measurement. This control knob is not available in the classical gain measurements.

In summary, we proposed a set of multidimensional spectroscopic measurements with nonclassical light. Quantum squeezing generated in FWM is used as a spectroscopy tool for the media where FWM is generated. By scanning the pump and probe frequencies, a 2D signal corresponding to the classical (gain) or quantum (NF) measurement reveals the internal structure of the multilevel quantum system. While gain measurements are sensitive to noise, squeezing is noise resistant with the balanced losses, which allows us to retain high spectral resolution of the measurement optical. The proposed spectroscopic technique applies to solid state systems, such as $\mathrm{SiV}^{-}$, as well as to the $\mathrm{GHz}$ spectral range and the gas phase, ${ }^{13}$ or other spectral 
regimes. ${ }^{28}$ Furthermore, while the nonlinear response can be investigated in single particle systems with high sensitivity, ${ }^{29}$ our main goal is to apply the FWM techniques in quantum sensors ${ }^{30}$ to study Raman transitions $^{31}$ of large biological macromolecules, where inhomogeneous broadening, environmental fluctuation effects, and a rich spectrum of internal degrees of freedom result in congested spectra, which are difficult to resolve. Combination of multiple FWMs in either cascading or $S U(1,1)$, which are routinely used to improve quantum squeezing, provides an additional control knob for improving spectral resolution of the multidimensional measurement and can further counteract internal losses. ${ }^{32}$ The proposed technique offers a class of measurements that are not limited to two-photon absorption and use different mechanisms of generation of quantum light not limited to the entangled two photon light sources.

See the supplementary material for the details of the microscopic calculations of the signals.

We would like to thank Professor Alexey Kalachev and Professor Philip Hemmer for the fruitful discussions. Z.Y., P.S., and K.E.D. gratefully acknowledge the support from the National Science Foundation of China (No. 11934011), the Zijiang Endowed Young Scholar Fund, the East China Normal University, and the Overseas Expertise Introduction Project for Discipline Innovation (111 Project, B12024). F.S. acknowledges financial support of the European Research Council under European Union's Seventh Framework Programme (No. FP7/2007-2013)/ERC Grant Agreement No. 319286 Q-MAC. S.M. gratefully acknowledges the support of the National Science Foundation through Grant No. CHE-1953045 and of the U.S. Department of Energy, Office of Science, Office of Basic Energy Sciences under Award No. DESC0020168. K.E.D. visits to UCI were sponsored by the DOE grant.

\section{DATA AVAILABILITY}

The data that support the findings of this study are available within this article and its supplementary material.

\section{REFERENCES}

${ }^{1}$ J. Nunn, I. Walmsley, M. Raymer, K. Surmacz, F. Waldermann, Z. Wang, and D. Jaksch, Phys. Rev. A 75, 011401 (2007).

${ }^{2}$ S. Moiseev and W. Tittel, New J. Phys. 13, 063035 (2011).

${ }^{3}$ X. Zhang, A. Kalachev, and O. Kocharovskaya, Phys. Rev. A 87, 013811 (2013).

${ }^{4}$ A. Kalachev, A. Berezhnoi, P. Hemmer, and O. Kocharovskaya, Laser Phys. 29, 104001 (2019).
${ }^{5^{5}}$. Takesue and K. Inoue, Phys. Rev. A 70, 031802 (2004).

${ }^{6}$ M. Levenson, R. Shelby, A. Aspect, M. Reid, and D. Walls, Phys. Rev. A 32, 1550 (1985).

${ }^{7}$ C. McCormick, V. Boyer, E. Arimondo, and P. Lett, Opt. Lett. 32, 178-180 (2007).

${ }^{8}$ C. Embrey, M. Turnbull, P. Petrov, and V. Boyer, Phys. Rev. X 5, 031004 (2015).

${ }^{9}$ S. L. Braunstein and P. Van Loock, Rev. Mod. Phys. 77, 513 (2005).

${ }^{10}$ V. Boyer, A. M. Marino, R. C. Pooser, and P. D. Lett, Science 321, 544-547 (2008).

${ }^{11}$ D.-K. Lim, K.-S. Jeon, H. M. Kim, J.-M. Nam, and Y. D. Suh, Nat. Mater. 9, 60-67 (2010).

${ }^{12}$ Z. Qin, L. Cao, H. Wang, A. Marino, W. Zhang, and J. Jing, Phys. Rev. Lett. 113, 023602 (2014).

${ }^{13}$ Y. Fang and J. Jing, New. J. Phys. 17, 023027 (2015).

${ }^{14} \mathrm{H}$. Wang, L. Cao, and J. Jing, Sci. Rep. 7, 40410 (2017).

${ }^{15}$ A. Dutt, K. Luke, S. Manipatruni, A. L. Gaeta, P. Nussenzveig, and M. Lipson, Phys. Rev. Appl. 3, 044005 (2015).

${ }^{16}$ K. E. Dorfman, F. Schlawin, and S. Mukamel, Rev. Mod. Phys. 88, 045008 (2016).

${ }^{17}$ F. Schlawin, K. E. Dorfman, and S. Mukamel, Acc. Chem. Res. 51, 2207-2214 (2018).

${ }^{18}$ S. Mukamel, M. Freyberger, W. P. Schleich, M. Bellini, A. Zavatta, G. Leuchs, C. Silberhorn, R. W. Boyd, L. S. Soto, A. Stefanov et al., J. Phys. B 53, 072002 (2020).

${ }^{19}$ Y. Michael, L. Bello, M. Rosenbluh, and A. Pe'er, npj Quantum Inf. 5, 81 (2019).

${ }^{20}$ M. Jasperse, L. Turner, and R. Scholten, Opt. Express 19, 3765-3774 (2011).

${ }^{21}$ Y.-W. Cho, K.-K. Park, J.-C. Lee, and Y.-H. Kim, Phys. Rev. Lett. 113, 063602 (2014).

${ }^{22}$ C. Weinzetl, J. Görlitz, J. N. Becker, I. A. Walmsley, E. Poem, J. Nunn, and C. Becher, Phys. Rev. Lett. 122, 063601 (2019).

${ }^{23}$ H. Sternschulte, K. Thonke, R. Sauer, P. C. Münzinger, and P. Michler, Phys. Rev. B 50, 14554-14560 (1994).

${ }^{24}$ A. Dietrich, K. D. Jahnke, J. M. Binder, T. Teraji, J. Isoya, L. J. Rogers, and F. Jelezko, New J. Phys. 16, 113019 (2014).

${ }^{25}$ D. Abramavicius, B. Palmieri, D. V. Voronine, F. Sanda, and S. Mukamel, Chem. Rev. 109, 2350-2408 (2009).

${ }^{26}$ V. Bužek, J. Mod. Opt. 37, 303-316 (1990).

${ }^{27}$ B. Yurke, S. L. McCall, and J. R. Klauder, Phys. Rev. A 33, 4033-4054 (1986).

${ }^{28}$ B. W. Adams, C. Buth, S. M. Cavaletto, J. Evers, Z. Harman, C. H. Keitel, A. Pálffy, A. Picón, R. Röhlsberger, Y. Rostovtsev, and K. Tamasaku, J. Mod. Opt. 60, 2-21 (2013).

${ }^{29}$ J. Obermeier, T. Schumacher, and M. Lippitz, "Nonlinear spectroscopy of plasmonic nanoparticles," Adv. Phys.: X 3, 1454341 (2018).

${ }^{30}$ B. J. Lawrie, P. D. Lett, A. M. Marino, and R. C. Pooser, ACS Photonics 6, 1307-1318 (2019).

${ }^{31}$ A. H. Hartog, Proc. SPIE 8421, 147-152 (2012).

${ }^{32}$ M. Manceau, G. Leuchs, F. Khalili, and M. Chekhova, Phys. Rev. Lett. 119, 223604 (2017). 